\title{
Effect of Dual Infection with Eimeria tenella and Subgroup J Avian Leukosis Virus on the Cecal Microbiome in Specific-Pathogen- Free Chicks
}

\author{
Ning Cui 1,2,3,4t, Xiuzhen Wang ${ }^{1,2+}$, Qi Wang ${ }^{5 \dagger}$, Hongmei Li $^{1,2}$, Fangkun Wang ${ }^{1,2}$ \\ and Xiaomin Zhao ${ }^{1,2 *}$ \\ 'Shandong Provincial Key Laboratory of Animal Biotechnology and Disease Control and Prevention, Shandong Agricultural \\ University, Tai'an, China, ${ }^{2}$ Shandong Provincial Engineering Technology Research Center of Animal Disease Control and \\ Prevention, Shandong Agricultural University, Tai'an, China, ${ }^{3}$ nstitute of Animal Science and Veterinary Medicine, Shandong \\ Academy of Agricultural Sciences, Jinan, China, ${ }^{4}$ Shandong Key Lab of Animal Disease Control and Breeding, Jinan, China, \\ ${ }^{5}$ Animal Husbandry and Veterinary Station of Xuzhou, Xuzhou, China
}

OPEN ACCESS

Edited by: Guillermo Tellez, University of Arkansas,

United States

Reviewed by:

Peter M. Rubinelli, University of Arkansas,

United States

Sherry Layton,

Vetanco, Argentina

*Correspondence:

Xiaomin Zhao

xmzhao66@163.com

tThese authors have contributed equally to this work.

Specialty section: This article was submitted to Veterinary Infectious Diseases,

a section of the journal Frontiers in Veterinary Science

Received: 18 July 2017 Accepted: 04 October 2017 Published: 25 October 2017

Citation:

Cui N, Wang X, Wang Q, Li H, Wang $F$ and Zhao $X$ (2017) Effect of Dual Infection with Eimeria tenella and Subgroup J Avian Leukosis Virus

on the Cecal Microbiome in Specific-Pathogen-Free Chicks.

Front. Vet. Sci. 4:177. doi: 10.3389/fvets.2017.00177
Understanding gut microflora alterations associated with gut parasites and other pathogens that drive these alterations may help to promote the understanding of intestinal flora's role in multiple-infected individuals. This study examined the effects of dual infection with Eimeria tenella and subgroup $\mathrm{J}$ avian leukosis virus (ALV-J) on the chick cecal microbiome. Specific-pathogen-free (SPF) chicks were infected with either ALV-J strain NX0101 at 1 day of age or E. tenella at 14 days of age, another group was infected with both pathogens. Cecal contents from chicks were extracted at the 21 days of age and examined using 16S rRNA genes illumina sequencing. A genus-level opportunistic pathogen enrichment and a decrease in possible resident probiotics were observed in response to all infection groups. Of note, E. tenella mainly induced a sharp decrease in the richness and diversity of cecal microflora from infected chicks because of the serious E. tenella-induced damage to intestinal tissues. ALV-J infection led to structural changes and increased the richness and diversity of the cecal microflora. As for $E$. tenella and ALV-J dual infected chicks, a marked enrichment of opportunistic pathogens in addition to some other bacteria that may play a role involving cecal microbiota carbohydrate transport and metabolic functions were also found compared to single pathogen-infected chicks. Overall, this study provides valuable insights into the SPF chick cecal microbial community, the modulations of this community in response to different pathogenic infections of single or dual infections, and the interactions between different pathogens and hosts from the perspective of intestinal microflora.

Keywords: dual infection, Eimeria tenella, subgroup J avian leukosis virus, 16S rRNA, cecal microflora

\section{INTRODUCTION}

The importance of understanding the dynamics of intestinal microbial ecology has been long recognized throughout the literature (1). The intestinal flora plays a vital role in harvesting energy from the diet, stimulating the proliferation of the intestinal epithelium, and developing the immune system in the host $(2,3)$. Regarding poultry, earlier studies from the cecum have 
demonstrated that this part of intestine harbors a complex microflora, and the absence of normal microflora in the cecum has been considered a major factor in the susceptibility of chicks to bacterial infection (4-6).

The gut microflora displays higher diversity and are maintained in a relative balance that is essential for host health, but is easily influenced by various diseases $(4,6,7)$. Coccidiosis is still one of the most endemic enteric diseases in broiler production (8-10). Coccidial stress has consistently been shown to sensitize broilers to enteritis, including necrotic enteritis (10). Infection of chickens with Eimeria tenella has stimulated the growth of Clostridium perfringens in both conventional and specificpathogen-free (SPF) birds (11-13). In addition, large numbers of Bacteroides sp., Enterobacteriaceae and some Streptococci, but low numbers of Lactobacilli and Bifidobacteria, have been seen in the ceca of infected chickens $(12,14)$. Leukemia in chickens, caused by the avian leukosis virus (ALV), has been reported over 100 years (15). J avian leukosis virus (ALV-J) was first isolated in 1988 from meat-type chickens in Great Britain (16). It induced mainly myelocytomatosis and nephromas (17) and could suppress growth of commercial broilers and SPF broilers $(18,19)$. The prevalence of ALV-J as one of the major diseases in laying hens has become a danger to the poultry industry, especially to the Chinese laying hens' industry in recent years. Intramuscular injection of ALV-J at 1 day of age induced a decreased number of Lactobacilli and Bifidobacteria, but increased the number of Escherichia coli and Enterococcus in the cecum 6 months postinfection (20).

A number of previous studies on poultry bacterial populations have relied on cultivation and enumeration of bacterial species (21), but most bacteria cannot easily be isolated from their habitats through the routine culturing methods used in most laboratories today. More recently, PCR-based culture-independent methods have been employed and $90 \%$ of the bacteria in the chicken gastrointestinal tract that represent previously unknown species were found using such techniques (22). Amplification of one or more hypervariable regions of the 16S rRNA region followed by parallel tag illumina sequencing is now commonly employed to analyze many different bacterial populations (23). A synergy between ALV-J and E. tenella that results in increasing pathogenesis in SPF chickens was underlined previously (24). To better characterize the interaction between different pathogens and the host from the perspective of intestinal flora, we have performed illumina sequencing of the V3 + V4 region of the $16 \mathrm{~S}$ rRNA genes using Ilumina Miseq PE300 sequencing to examine and analyze the composition of gut microflora in the chick ceca under single or dual infection with E. tenella and ALV-J. Except that common features of cecal microflora were observed in both pathogen infections, distinctive bacteria community characteristics in response to different pathogens of single or dual infections were also shown in our study.

\section{MATERIALS AND METHODS}

\section{Coccidium and Virus}

The wild type E. tenella strain SD-01 was stored in our laboratory (25). Sporulated oocysts were stored in $2.5 \%$ potassium dichromate at $4^{\circ} \mathrm{C}$ and propagated in 3 weeks old chickens every 6 months as previously described (26). The sporulated oocysts for the experiments were purified from newly infected chickens.

The ALV-J field strain NX0101 was isolated from a meat-type parent breeder farm by our lab in Ningxia province of China in 2001 (27). Chicken DF-1 cell line cultured in Dulbecco's modified Eagle's medium (DMEM) supplemented with 10\% fetal bovine serum (FBS) was used for virus culturing (kept in our laboratory). DF-1 cells were infected with NX0101 until cells grew about $90 \%$ confluence and maintained in DMEM supplemented with $1 \% \mathrm{FBS}$ in $37^{\circ} \mathrm{C}$ and $5 \% \mathrm{CO}_{2}$ after infection. Newly propagated virus was titered as the $50 \%$ tissue culture infective dose $\left(\mathrm{TCID}_{50}\right) \mathrm{ml}^{-1}$ using the Reed-Muench formula directed by ELISA (28).

\section{Experiment Design}

The study protocol and all animal studies were approved by the Shandong Agricultural University Animal Care and Use Committee (SACUC Permission number: AVM140301-19).

Specific-pathogen-free chicks (Dongyue poultry, Taian, China) were used for the infection experiments. One-day-old male SPF chicks were randomly divided into four groups of 15 birds each. They were inoculated in the abdomen with ALV-J of $10^{-3.5} \mathrm{TCID}_{50}$ at 1 day of age, challenged orally with E. tenella of 6,000 sporulated oocysts at 14 days of age, or both. The control group were inoculated or challenged orally with PBS. Chicks were housed in separate pens in the same building at the Research Animal Facility at Shandong Agricultural University and provided with coccidiostat-free feed and water ad libitum. Cecal samples were aseptically collected 7 days post-infection from three chicks randomly selected from each group and immediately stored at $-80^{\circ} \mathrm{C}$ until DNA extraction.

\section{DNA Extraction}

The contents and the mucosal wall of the cecal samples from each chick were homogenated, and DNA was extracted from pooled samples using the E.Z.N.A Soil DNA Kit (OMEGA) following the manufacturer's instructions. Samples were measured by $1 \%$ agarose gel electrophoresis to assess integrity and with a Nanodrop ND-1000 spectrophotometer (Thermo Scientific) to assess DNA quantity.

\section{S rRNA Amplification and Miseq Sequencing}

Preliminary tests of PCR amplification for the V3 + V4 hypervariable region of $16 \mathrm{~S}$ rRNA gene were carried out in a $20 \mu \mathrm{l}$ reaction containing $1 \times$ PCR buffer (containing $2 \mathrm{mM} \mathrm{MgCl} 2$ ), $0.25 \mathrm{mM}$ each dNTP,0.4mMeach primer, 1UTransStartFastPfuDNAPolymerase (TransGen AP221-02), and 10 ng DNA template. The primers used were 5'-ACTCCTACGGGAGGCAGCA-3' with barcode (forward primer: 338F) and 5'-GGACTACHVGGGTWTCTAAT-3' with barcode (reverse primer: 806R). PCR products were assessed with $2 \%$ agarose gel electrophoresis. PCR products of the same sample were assembled within a PCR tube and excised from a $2 \%$ agarose gel stained with ethidium bromide. Purification was 
performed using the AxyPrepDNA Gel Extraction Kit (AXYGEN) and eluted with Tris-HCl. DNA quality was assessed by $2 \%$ agarose gel electrophoresis, and the concentration was assessed by the QuantiFluor ${ }^{\mathrm{TM}}$-ST blue fluorescence quantitative system (Promega). Barcoded samples were combined equal concentrations according to volume of sequencing. Illumina sequencing was performed commercially at Shanghai Majorbio Biopharm Biotechnology Co., Ltd., on a MiSeq Sequencer platform. ${ }^{1}$

\section{Bioinformatic Analysis of Sequencing Data}

PE reads obtained from Miseq sequencing were spliced according to overlap relationships. The quality of the sequence was conducted with quality control and filtration to obtain highquality sequences. The sequences from all the samples were clustered into operational taxonomic units (OTUs) based on a 97\% identity threshold by Uparse version 7.1. ${ }^{2}$ The most abundant sequence of each OTU ( $97 \%$ similarity) was BLAST searched against the Silva databases (Release $115^{3}$ ) to determine the phylogeny of the OTU. A variety of diversity index analysis and detection of sequencing depth were carried out based on OUT and OUT clustering results. Community structure in each classification level was analyzed based on taxonomists analysis. Venn diagrams and heat maps were performed between groups with an OTU definition at a similarity cutoff of $97 \%$ $(29,30)$. Function abundance profiles of cecal microbiota were established according to OTU abundance of different groups standardized using PICRUSt software, and their functional information was searched against the eggNOG database (evolutionary genealogy of genes: non-supervised Orthologous Groups ${ }^{4}$.

\section{Statistical Analyses}

Differences between populations had been analyzed using parametric (ANOVA with the Tukey's multiple comparison test) and nonparametric statistical methods. All results are presented as the mean value $( \pm \mathrm{SE})$. Differences between groups were declared significant at $p<0.05$.

\section{RESULTS}

\section{Cecal Pathology Caused by E. tenella Infection}

Both E. tenella single- and dual-infected chickens showed serious damage in the ceca, with coagulation necrosis, thickening of the mucosa, and edematous swelling (Figure 1). The ALV-J infected chicks and the uninfected controls showed no obvious pathology.

${ }^{4} \mathrm{http} / / /$ eggnog.embl.de/
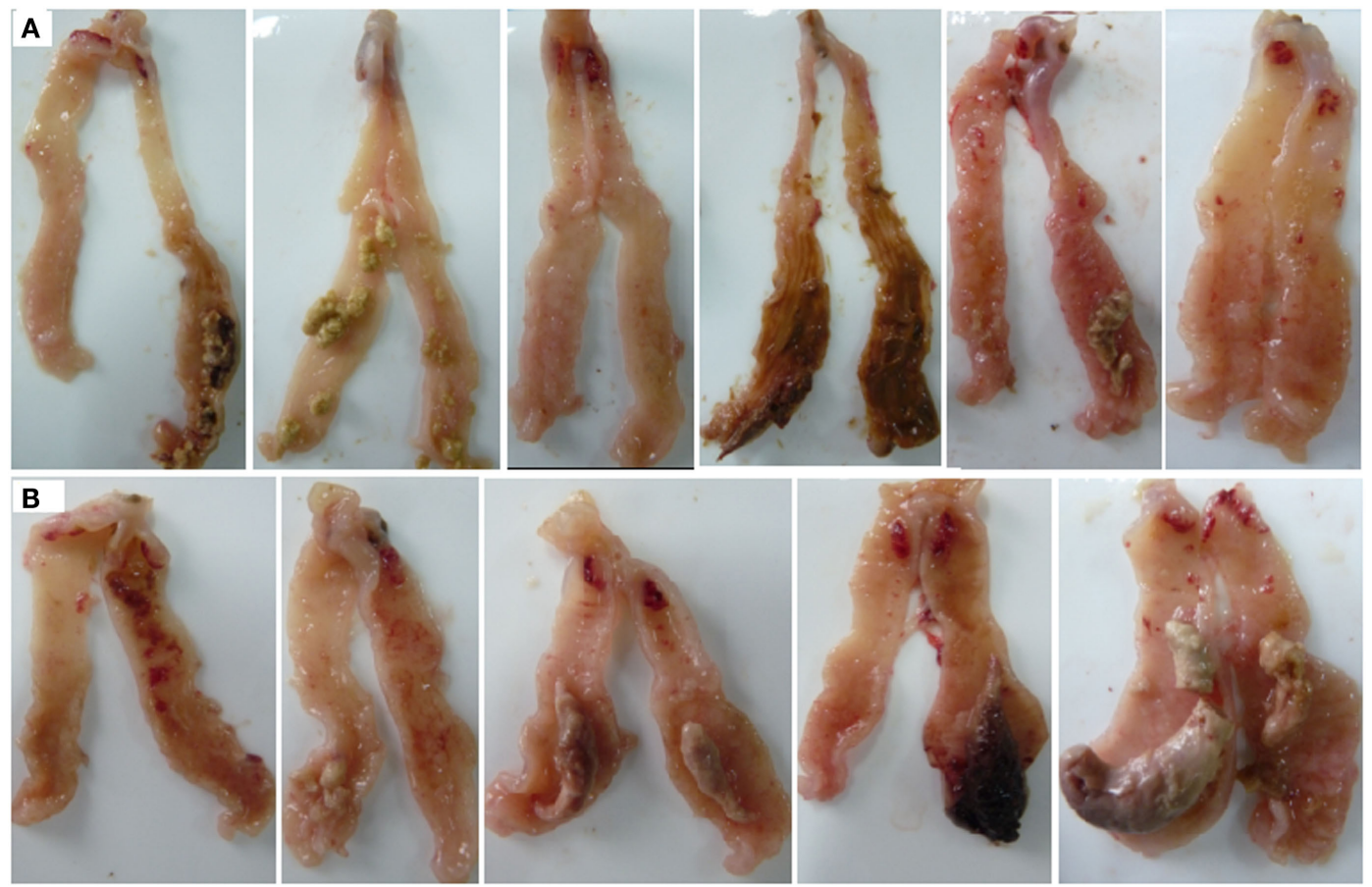

FIGURE 1 | Gross lesions in the ceca of Eimeria tenella-infected chicks. (A) Chickens infected with E. tenella. and E. tenella. 


\section{Quality Test for gDNA and PCR Products of 165 rRNA}

The gDNA of each sample was above $3 \mathrm{~kb}$ with slight protein, pigment, and other pollution of impurities. The concentration was higher than $300 \mathrm{ng} / \mu \mathrm{l}$, which was conformed to the experimental requirements. PCR was attempted to perform subsequent experiments. PCR products of $16 \mathrm{~S}$ rRNA using $338 \mathrm{~F}$ and $806 \mathrm{R}$ were $437 \mathrm{bp}$ with the concentration higher than $10 \mathrm{ng} / \mu \mathrm{l}$ were used for subsequent sequencing.

\section{General Comparisons of 16S rRNA Reads Using OTU Analysis}

The 16S rRNA sequence reads were binned according to their sequence similarities with one another and independent of any database hits or searches. After pre-processing, 360,388 highquality sequences were obtained for subsequent bioinformatics analyses. With an OTU definition at a similarity cutoff of $97 \%$, a total of 381 OTUs were identified among the four different groups examined with 306, 131, 323, and 219 OTUs for the

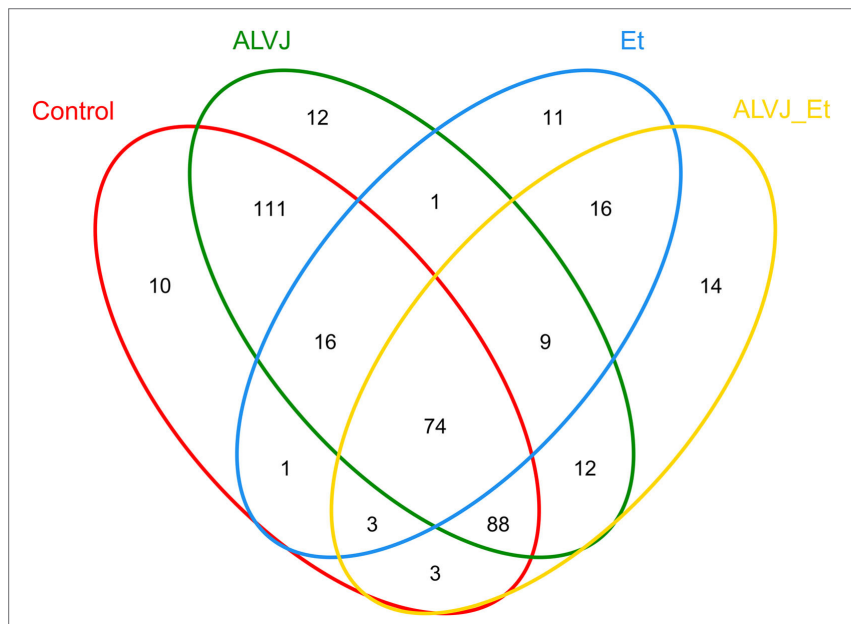

FIGURE 2 | Venn diagram illustrating shared and unique operational taxonomic units (OTUs) from different infectious groups. Numbers below groups indicate the number of OTUs within each sector. control group, E. tenella, and ALV-J single- or dual-infected groups, respectively. As shown in Figure 2, different infection groups showed diverse OTU patterns. This was also reflected by the abundance index (Ace and Chao) and the diversity index (Shannon and Simpson) analyses, which suggested that sample richness and diversity differed between the different groups (Table 1). These indexes preliminarily indicated that $E$. tenella sharply decreased the diversity and richness of cecal flora, and that ALV-J infection induced growth of some bacteria than the control group. Cecal flora diversity and richness of dual-infected chickens showed a slight decrease when compared to the control ones.

\section{Changes in Cecal Flora over Different Groups at Different Levels}

The microflora and compositions of the four groups were analyzed and compared through the relative abundance of OTUs. Each of the 381 OTUs were analyzed for significant enrichment or depletion in different groups. Then, OTUs with significant changes $(p>0.05)$ were sorted by abundance and classified using Silva. A number of OTUs were uniformly affected across groups.

The overall microbial composition for each group at the phylum level is shown in Figure 3A. When bacteria in normal chick cecum at 21 days of age were identified, they belonged predominantly to the Firmicutes (67.12\%), with Bacteroidetes (26.2 6\%), Proteobacteria (3.44\%), Cyanobacteria (2.67\%), and Tenericutes $(0.49 \%)$ also identified. The structure of the cecal flora was in disorder in both single- and dualinfected groups. We demonstrated a significant increase of Proteobacteria and a slight decrease of Bacteroidetes in ALV-J infected chickens. Proteobacteria were found to be significantly enriched, while members of Bacteroidetes, Cyanobacteria, and Tenericutes were observed with a marked decrease in both E. tenella single- and dual-infected chickens. Chickens that were dual-infected with ALV-J and E. tenella showed proliferation of some bacteria like Planococcaceae and Bacillaceae in different chickens. Furthermore, a significant enrichment of Lachnospiraceae, Enterobacteriaceae, and Enterococcaceae, with the complete depletion of Ruminococcaceae, Rikenellaceae,

TABLE 1 | Number of OTUs per groups and estimators of sequence diversity and richness.

\begin{tabular}{|c|c|c|c|c|c|c|}
\hline Sample ID & No. of reads & No. of OTUs & Ace (richness) & Chao1 (richness) & Shannon (diversity) & Simpson (diversity:1-D) \\
\hline Control 1 & 16,883 & 288 & 309 (299, 329) & $305(295,328)$ & 0.997986 & $4.23(4.21,4.25)$ \\
\hline Control 2 & 16,883 & 280 & $292(285,306)$ & $295(286,320)$ & 0.998519 & $4.06(4.04,4.09)$ \\
\hline Control 3 & 16,883 & 277 & $293(285,309)$ & $297(285,325)$ & 0.998223 & $4(3.97,4.02)$ \\
\hline ALV-J 1 & 16,883 & 295 & $317(307,338)$ & $317(305,345)$ & 0.997868 & $4.34(4.32,4.36)$ \\
\hline ALV-J 2 & 16,883 & 285 & $314(301,339)$ & $308(296,336)$ & 0.997572 & $4.2(4.18,4.22)$ \\
\hline ALV-J 3 & 16,883 & 290 & $305(297,322)$ & $304(296,325)$ & 0.998282 & $4.3(4.28,4.32)$ \\
\hline E. tenella 1 & 16,883 & 112 & $182(157,222)$ & $143(126,180)$ & 0.997631 & $1.06(1.04,1.08)$ \\
\hline E. tenella 2 & 16,883 & 49 & $96(73,139)$ & $68(55,111)$ & 0.998993 & $1.73(1.71,1.75)$ \\
\hline E. tenella 3 & 16,883 & 49 & $107(80,156)$ & $71(56,117)$ & 0.998934 & $1.26(1.24,1.27)$ \\
\hline ALV-J + E. tenella 1 & 16,883 & 116 & $195(166,240)$ & $164(137,226)$ & 0.997808 & $2.41(2.38,2.43)$ \\
\hline ALV-J + E. tenella 2 & 16,883 & 190 & $214(202,238)$ & $233(208,293)$ & 0.997986 & $3.09(3.07,3.12)$ \\
\hline ALV-J + E. tenella 3 & 16,883 & 121 & $159(140,194)$ & $156(136,201)$ & 0.997868 & $1.73(1.71,1.75)$ \\
\hline
\end{tabular}

OTUs, operational taxonomic units; ALV-J, subgroup J avian leukosis virus; E. tenella, Eimeria tenella. 

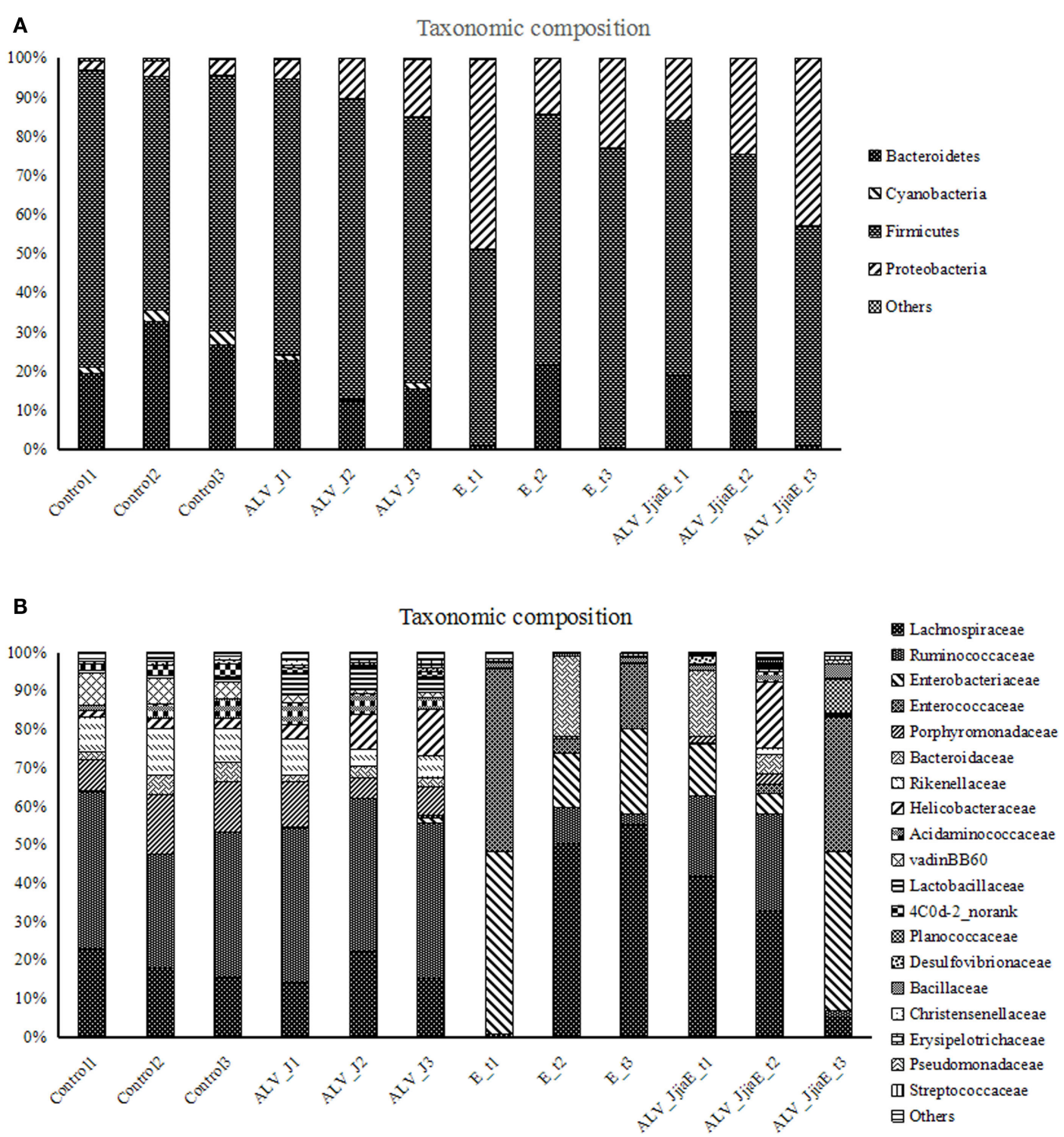

FIGURE 3 | Relative contributions of dominant phyla (A) and family (B) in the cecal microbiota using V3-V4 amplicon sequencing $(n=360,388)$.

and Porphyromonadaceae, was also observed in dual-infected chickens at the family level (Figure 3B).

Genus distributions were also analyzed among different groups (Figure 4). The dominant identified genera in normal SPF chicks were Lachnospiraceae_unclassified (15.99\%), Ruminococcaceae_uncultured (9.73\%), Faecalibacterium (8.26\%), Barnesiella (8.14\%), Helicobacter (7.12\%), Rikenella (4.36\%), Lachnospiraceae_uncultured (4.11\%), Bacteroides (4.08\%), and Ruminococcaceae_incertae_sedis (4.01\%). ALV infection led to the enrichment of Anaerofilum, Subdoligranulum, Lactobacillus, Lactococcus, Enterococcus, Klebsiella, Escherichia-Shigella, Akkermansia, Helicobacter, and members of Bacillales and Erysipelotrichaceae, with the depletion of Shuttleworthia, Ruminococcus, Faecalibacterium, Rikenella and members of Bacteroidaceae and Porphyromonadaceae. E. tenella infection caused a dramatic change in the structure of the cecal microflora, characterized by a pronounced depletion of resident flora and enrichment of a large number of conditioned pathogenic bacteria, including Escherichia-Shigella, Enterococcus, Bacillus, Staphylococcus, etc. Chickens dual-infected with ALV and E. tenella showed a diverse structure of cecal microflora and mainly induced proliferation of certain bacteria, such as Lysinibacillus, which is dominant of Planococcaceae and Bacillus from Bacillaceae, as well as a marked enrichment of opportunistic pathogens like Escherichia-Shigella and Bacillus, etc.

\section{Function Abundance Profile of Cecal Microbiota from All Groups}

Function abundance profiles of the cecal microbiota of different groups were evaluated based on OTUs (Figure 5). 


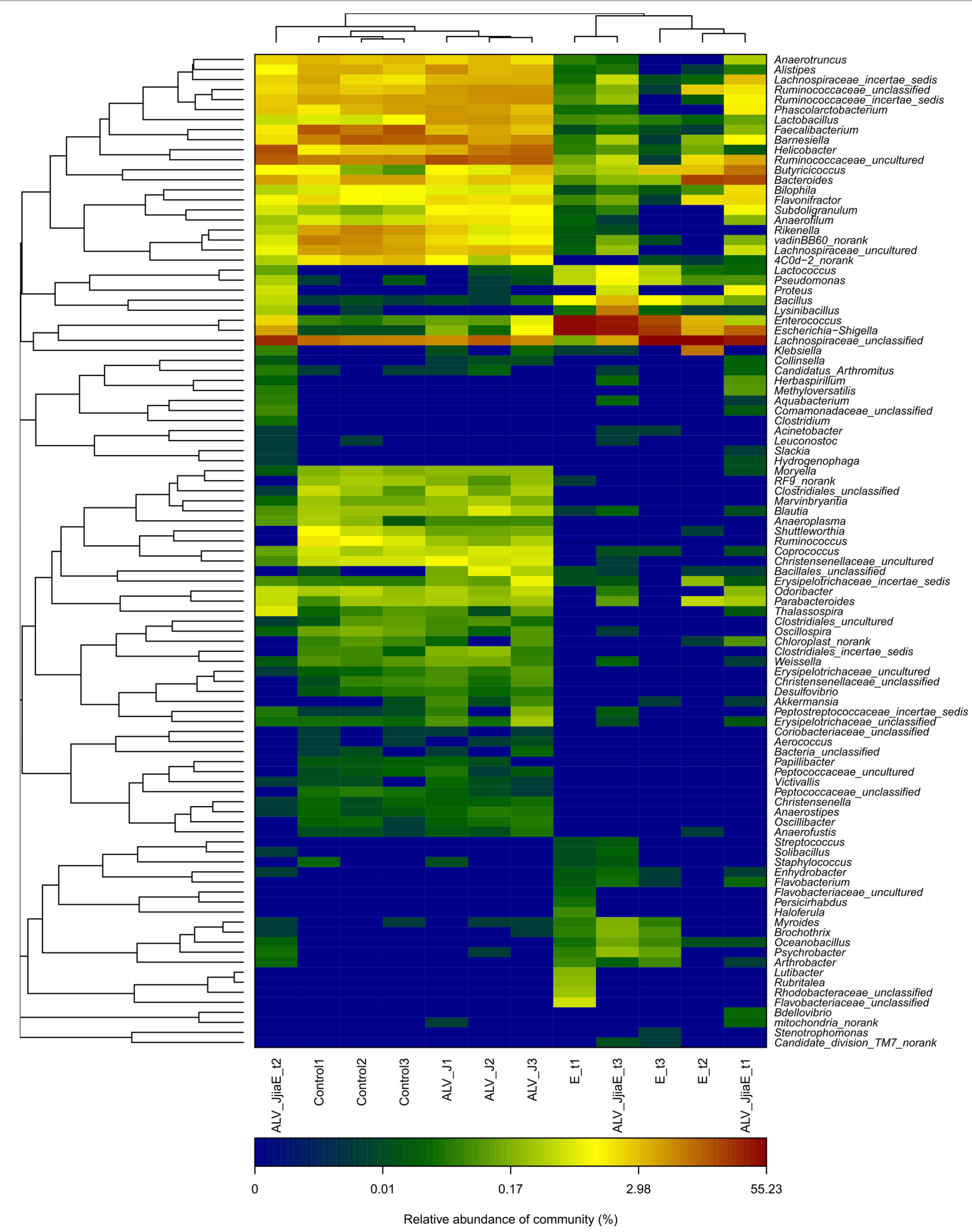

FIGURE 4 | The heatmap of top 100 genera between different groups. Double hierarchical dendrogram shows the bacterial distribution. The heatmap plot depicts the relative percentage of each bacterial genus within each sample. The relative values for bacterial family are indicated by color intensity with the legend indicated under the heatmap. 


\section{COG Function Classification}

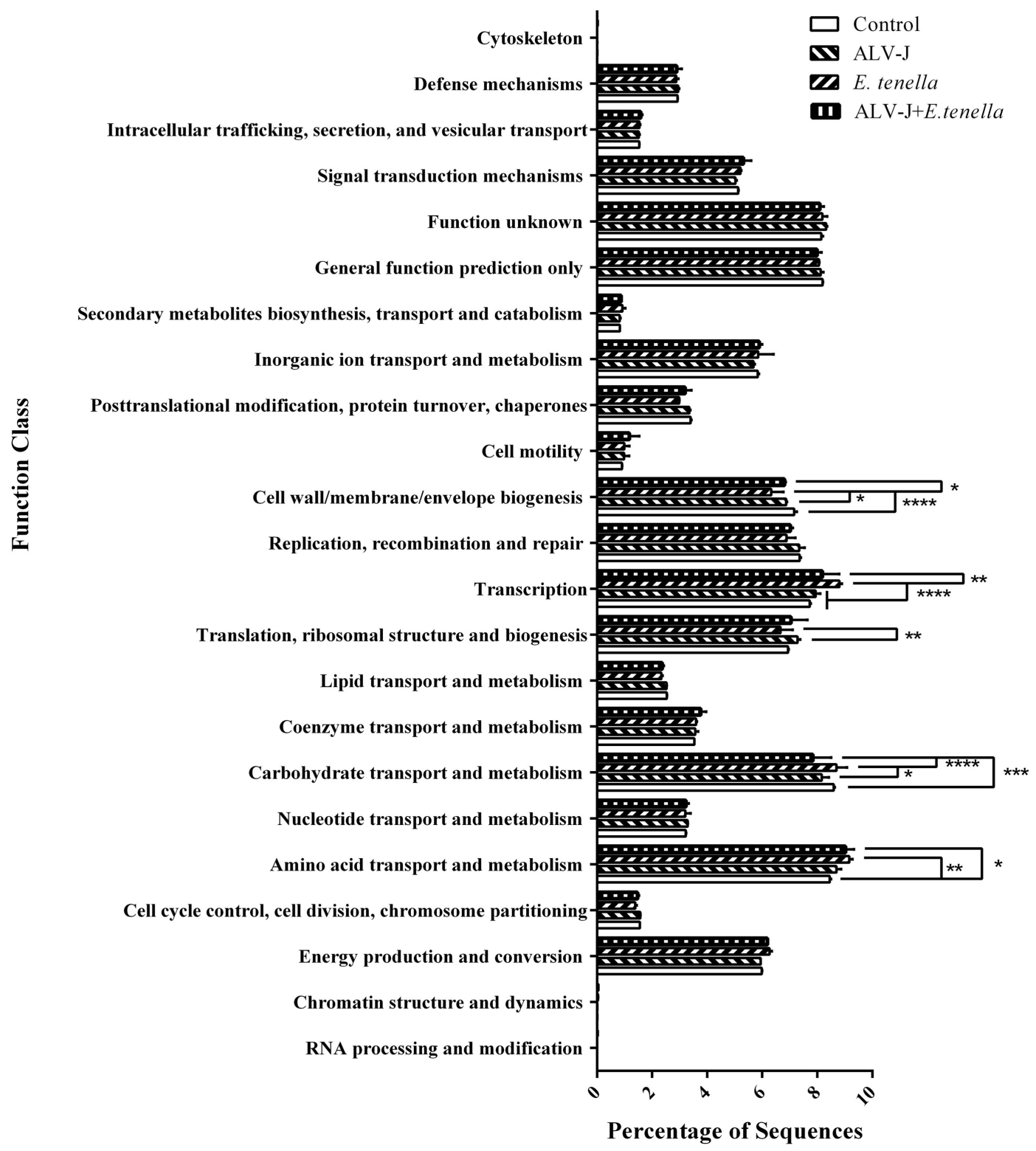

FIGURE 5 | Function abundance profile of cecal microbiota of different groups. COG, clusters of orthologous groups of proteins. Error bars represent SDs. ${ }^{*}$ Statistically significant difference between groups $(p<0.05)$.

Genes that take part in translation, ribosomal structure, and biogenesis were significantly enriched in cecal microbiota of ALV-J-infected chickens, and genes that play a role in carbohydrate transport and metabolism were found to be significantly reduced, whereas $E$. tenella infection enhanced the function of amino acid transport and metabolism, carbohydrate transport and metabolism and transcription of cecal microbiota. In addition to the decrease of the translation, ribosomal structure and biogenesis role of cecal microbiota that are similar to ALV-J infected chickens, genes that take part in cell wall/membrane/ envelope biogenesis were also found to be sharply reduced.
Dual infection of E. tenella further aggravated the reduced carbohydrate transport and metabolism function of cecal microbiota caused by ALV-J infection. However, the function of cell wall/membrane/envelope biogenesis and translation, ribosomal structure, and biogenesis in dual-infected chickens recovered, to some extent.

\section{DISCUSSION}

Investigation on gut microflora contributes to a greater understanding of the pathogenic mechanism of many pathogens. In 
the present study, we compared cecal microbial changes in chicks that were single- or dual-infected with E. tenella and ALV-J, and marked differences were observed.

Eimeria tenella caused serious cecal pathological changes on 7 days post-infection in our study. A sharp decrease in richness and diversity of cecal microflora with uniform depletion of a number of genera from infected chicks were observed as a result of the serious damage of intestinal tissues caused by E. tenella infection. A reduction of potentially beneficial bacteria was detected, as previously reported $(12,14)$. The abundance of members of Ruminococcaceae using sticky protein as a food source, Anaeroplasma adhered on the cell surface, and Phascolarctobacterium, Faecalibacterium, Coprococcus, Ruminococcus, and Blautia which are helpful for improving production of short chain fatty acids, were also reduced. These bacteria have two aspects of antimicrobial effects: through their own engraftment antagonism and through the produced acetate as the main product of its metabolism and materials with broad-spectrum antimicrobial activity to reduce the intestinal local $\mathrm{pH}$, which is helpful for inhibiting conditional pathogenic bacteria and reducing the production of harmful substances like endotoxin $(21,31)$. Thus, gut microbe probiotics may influence the immune system directly or indirectly by promoting metabolites with positive effects on the immune system. In addition, Subdoligranulum, a relatively abundant bacteria of Clostridiales in chicken cecum (32), was sharply reduced after E. tenella infection. The clostridia subgroup comprises several strictly anaerobic, butyrate-producing species. Butyrate plays an important role in animal health by regulating the immune system and reducing chronic inflammation. Reduction of butyrate may lead to high levels of chronic inflammation and immune disorders (33). Thus, Subdoligranulum, reduced in E. tenella-infected chicks, which is related to butyrate production, is possibly involved in the enteritis associated with coccidiosis. In contrast, the abundance of many opportunistic pathogens, like Escherichia-Shigella and Enterococcus in the cecum of E. tenella-infected chicks, were changed, in previous studies using culture-based methods $(11,12)$. Furthermore, many other opportunistic pathogens such as Bacillus, Klebsiella, and Proteus were also enriched. Metabolites decomposed by these bacteria are toxic for intestinal epithelial cells and can cause or exacerbate enteritis. E. tenella infection causes serious intestinal mucosal injuries that are harmful for planting and growth of resident bacteria, thus leading to decreased richness and diversity of cecal microflora, which may further aggravate the cecal lesions and increase the risk of secondary infection.

J avian leukosis virus (strain NX0101) begins to replicate quickly about 7 days post-infection and reaches a peak 17 days post-infection $(24,34,35)$. Human immunodeficiency virus (HIV), another single-stranded positive-sense RNA alpharetrovirus, which is similar to ALV-J, was reported to induce disorders of gut microflora in the early phase of infection (36). Cecal microflora of ALV-J-infected chicks were studied 21 days postinfection: the richness and diversity increased and the microflora structure was greatly changed compared to uninfected chicks in the present study. Similar to E. tenella infection, the abundance of potentially beneficial bacteria (Rikenella, Ruminococcus, and Faecalibacterium) were reduced. Resident flora stimulates the immune function of the host to mature as antigens. Reduced abundance of these bacteria weakens the intestinal immune function and cause enrichment of conditioned pathogens, such as Escherichia-Shigella, Helicobacter, and members of Enterobacteriaceae. It is possible that ALV-J-induced immunosuppression via changing the structure of intestinal flora and caused a high rate of secondary infection (37). In addition, ALV-J infection increased the richness and diversity of cecal microflora by enrichment of genera that were not detectable in uninfected chicks, such as Akkermansia, Klebsiella, etc. Ectopic of gut bacteria and their products expelled into the host's circulatory system are associated with certain chronic immune activation $(38,39)$. Chronic immune activation status helps the increase of HIV virus load in the host $(40,41)$. The structural changes of cecal microflora might contribute to replication of ALV-J in gut and, thus, the key factors of the pathogenicity of ALV chronic infection.

We have demonstrated that many bacterial genera, not just a select few, are responsible for the overall difference in cecal microflora associated with single or dual infection. Abundance variation of many bacteria belonging to the same phylum level is not consistent. No obvious synergistic or additive effects were detected in the above bacteria in dual-infected chicks, indicating that these bacteria may play little role in the pathogenicity caused by dual infection with E. tenella and ALV-J. However, it is worth to note that dual infection of E. tenella and ALV-J induced proliferation of some bacteria like Lysinibacillus, which is dominant of Planococcaceae, and Bacillus from Bacillaceae, etc. Furthermore, dual infection of E. tenella further aggravated the reduced carbohydrate transport and metabolism function of cecal microbiota caused by ALV-J infection. Thus, it is urgent to study the possible role of these proliferated bacteria in regulating the functional abundance profile performed by cecal microbiota, which might be of vital importance in revealing the pathology of multiply infected individuals.

In conclusion, both single or dual infection with E. tenella and ALV-J lead to obvious changes in cecal microflora of SPF chickens. We have identified several common changes of bacterial genera contributing to different pathogen-induced differences in the overall gut microbial community, with rapid alteration from protective species to those that promote inflammation and cause diseases. E. tenella causes serious injuries to cecal structure and decreases the richness and diversity of cecal microflora. Conversely, ALV-J tends to develop a chronic immune activation micro-environment in favor of viral replication by more varieties of bacteria. Dual infection with E. tenella and ALV-J mainly caused proliferation of some bacteria compared to single-infected chicks and may play a role involving carbohydrate transport and metabolism function of cecal microbiota. Collectively, we can conclude that a significant difference in cecal bacterial microflora exists between different infected groups. Further researches are needed to elucidate the mechanism of dual infection on gut microflora and its interaction with the host. 


\section{ETHICS STATEMENT}

The study protocol and all animal studies were approved by the Shandong Agricultural University Animal Care and Use Committee (SACUC Permission number: AVM140301-19).

\section{AUTHOR CONTRIBUTIONS}

NC, XW, and QW: collection and assembly of the data, manuscript writing, and data analysis; NC and XW: discussion and

\section{REFERENCES}

1. Savage DC. Microbial ecology of the gastrointestinal tract. Annu Rev Microbiol (1977) 31:107-33. doi:10.1146/annurev.mi.31.100177.000543

2. Hooper LV, Wong MH, Thelin A, Hansson L, Falk PG, Gordon JI. Molecular analysis of commensal host-microbial relationships in the intestine. Science (2001) 291:881-4. doi:10.1126/science.291.5505.881

3. Kelly D, Conway S, Aminov R. Commensal gut bacteria: mechanisms of immune modulation. Trends Immunol (2005) 26:326-33. doi:10.1016/j. it.2005.04.008

4. Barnes EM. The intestinal microflora of poultry and game birds during life and after storage. J Appl Bacteriol (1979) 46:407-19. doi:10.1111/j.1365-2672.1979. tb00838.x

5. Mead GC, Adams BW. Some observation on the caecal microflora of the chick during the first two weeks of life. Br Poult Sci (1975) 16:169-76. doi:10.1080/00071667508416174

6. Zhu XY, Zhong T, Pandya Y, Joerger RD. 16S rRNA-based analysis of microbiota from the cecum of broiler chickens. Appl Environ Microbiol (2002) 68:124-37. doi:10.1128/AEM.68.1.124-137.2002

7. Gong J, Si W, Forster RJ, Huang R, Yu H, Yin Y, et al. 16S rRNA gene-based analysis of mucosa-associated bacterial community and phylogeny in the chicken gastrointestinal tracts: from crops to ceca. FEMS Microbiol Ecol (2007) 59:147-57. doi:10.1111/j.1574-6941.2006.00193.x

8. Liu Y, Zheng J, Li J, Gong P, Zhang X. Protective immunity induced by a DNA vaccine encoding Eimeria tenella rhomboid against homologous challenge. Parasitol Res (2013) 112:251-7. doi:10.1007/s00436-012-3132-2

9. Zhang DF, Xu H, Sun BB, Li JQ, Zhou QJ, Zhang HL, et al. Adjuvant effect of ginsenoside-based nanoparticles (ginsomes) on the recombinant vaccine against Eimeria tenella in chickens. Parasitol Res (2012) 110:2445-53. doi:10.1007/s00436-011-2784-7

10. Williams RB. Intercurrent coccidiosis and necrotic enteritis of chickens: rational, integrated disease management by maintenance of gut integrity. Avian Pathol (2005) 34:159-80. doi:10.1080/03079450500112195

11. Bradley RE, Radhakrishnan CV. Coccidiosis in chickens: obligate relationship between Eimeria tenella and certain species of cecal microflora in the pathogenesis of the disease. Avian Dis (1973) 17:461-76. doi: $10.2307 / 1589145$

12. Kimura N, Mimura F, Nishida S, Kobayashi A. Studies on the relationship between intestinal flora and cecal coccidiosis in chicken. Poult Sci (1976) 55:1375-83. doi:10.3382/ps.0551375

13. Turk DE, Littlejohn VP. Coccidial infections and gut microflora. Poult Sci (1987) 66:1466-9. doi:10.3382/ps.0661466

14. Fukata T, Kageyama A, Baba E, Arakawa A. Effect of infection with Eimeria tenella upon the cecal bacterial population in monoflora chickens. Poult Sci (1987) 66:841-4. doi:10.3382/ps.0660841

15. Meyers P, Dougherty RM. Immunologic reactivity to viral antigens in chickens infected with avian leukosis viruses. J Natl Cancer Inst (1971) 46:701-11.

16. Payne LN. Retrovirus-induced disease in poultry. Poult Sci (1998) 77:1204-12. doi:10.1093/ps/77.8.1204

17. Payne LN, Brown SR, Bumstead N, Howes K, Frazier JA, Thouless ME. A novel subgroup of exogenous avian leukosis virus in chickens. J Gen Virol (1991) 72:801-7. doi:10.1099/0022-1317-72-4-801

18. Landman WJ, Post J, Boonstra-Blom AG, Buyse J, Elbers AR, Koch G. Effect of an in ovo infection with a Dutch avian leukosis virus subgroup J isolate on manuscript revision; XZ, HL, and FW: concept and design, data analysis, manuscript revision, and final approval of the manuscript.

\section{FUNDING}

This work was supported by a grant from the National Natural Science Foundation of China (No. 31572514) and Funds of Shandong "Double Tops Program."

the growth and immunological performance of SPF broiler chickens. Avian Pathol (2002) 31:59-72. doi:10.1080/03079450120106633

19. Stedman N, Brown T. Body weight suppression in broilers naturally infected with avian leukosis virus subgroup J. Avian Dis (1999) 43:604-10. doi: $10.2307 / 1592664$

20. Huang JQ. Mixed infection of avian leukosis virus and salmonella pullorum. Ph.D. thesis. Tai'an, China: Shandong Agricultural University (2013).

21. Biggs P, Parsons CM. The effects of several organic acids on growth performance, nutrient digestibilities, and cecal microbial populations in young chicks. Poult Sci (2008) 87:2581-9. doi:10.3382/ps.2008-00080

22. Handelsman J. Metagenomics: application of genomics to uncultured microorganisms. Microbiol Mol Biol Rev (2004) 68:669-85. doi:10.1128/ MMBR.68.4.669-685.2004

23. Sogin ML, Morrison HG, Huber JA, Mark Welch D, Huse SM, Neal PR, et al. Microbial diversity in the deep sea and the underexplored "rare biosphere". Proc Natl Acad Sci U S A (2006) 103:12115-20. doi:10.1073/pnas.0605127103

24. Cui N, Wang Q, Shi W, Han L, Wang J, Ma X, et al. Synergy of subgroup J avian leukosis virus and Eimeria tenella to increase pathogenesis in specific-pathogen-free chickens. Vet Immunol Immunopathol (2016) 2016(177):42-7. doi:10.1016/j.vetimm.2016.06.001

25. Sun H, Wang L, Wang T, Zhang J, Liu Q, Chen P, et al. Display of Eimeria tenella EtMic2 protein on the surface of Saccharomyces cerevisiae as a potential oral vaccine against chicken coccidiosis. Vaccine (2014) 32:1869-76. doi:10.1016/j. vaccine.2014.01.068

26. Fetterer RH, Barfield RC. Characterization of a developmentally regulated oocyst protein from Eimeria tenella. J Parasitol (2003) 89:553-64. doi:10.1645/ GE-3159

27. Cui Z, Du Y, Zhang Z, Silva R. Comparison of Chinese field strains of avian leukosis subgroup J viruses with prototype strain HPRS-103 and United States strains. Avian Dis (2003) 47:1321-30. doi:10.1637/6085

28. Reed LJ, Muench H. A simple method of estimating fifty percent endpoints. Am J Hygiene (1938) 27:4932497.

29. Cole JR, Wang Q, Cardenas E, Fish J, Chai B, Farris RJ, et al. The Ribosomal Database Project: improved alignments and new tools for rRNA analysis. Nucleic Acids Res (2009) 37:D141-5. doi:10.1093/nar/gkn879

30. Eisen MB, Spellman PT, Brown PO, Botstein D. Cluster analysis and display of genome-wide expression patterns. Proc Natl Acad Sci U S A (1998) 95:14863-8. doi:10.1073/pnas.95.25.14863

31. Baba E, Fukata T, Arakawa A. Factors influencing enhanced Salmonella typhimurium infection in Eimeria tenella-infected chickens. Am J Vet Res (1985) 46:1593-6.

32. Bjerrum L, Engberg RM, Leser TD, Jensen BB, Finster K, Pedersen K. Microbial community composition of the ileum and cecum of broiler chickens as revealed by molecularand culture-based techniques. Poult Sci (2006) 85:1151-64. doi:10.1093/ps/85.7.1151

33. Lund M, Bjerrum L, Pedersen K. Quantification of Faecalibacterium prausnitzii- and Subdoligranulum variabile-like bacteria in the cecum of chickens by real-time PCR. Poult Sci (2010) 89:1217-24. doi:10.3382/ps.2010-00653

34. Gao Y, Liu Y, Guan X, Li X, Yun B, Qi X, et al. Differential expression of immune-related cytokine genes in response to J group avian leukosis virus infection in vivo. Mol Immunol (2015) 64:106-11. doi:10.1016/j. molimm.2014.11.004

35. Wang F, Wang X, Chen H, Liu J, Cheng Z. The critical time of avian leukosis virus subgroup J-mediated immunosuppression during early stage infection 
in specific pathogen-free chickens. J Vet Sci (2011) 12:235-41. doi:10.4142/ jvs.2011.12.3.235

36. Gori A, Tincati C, Rizzardini G, Torti C, Quirino T, Haarman M, et al. Early impairment of gut function and gut flora supporting a role for alteration of gastrointestinal mucosa in human immunodeficiency virus pathogenesis. J Clin Microbiol (2008) 46:757-8. doi:10.1128/JCM.01729-07

37. Dong X, Zhao P, Chang S, Ju S, Li Y, Meng F, et al. Synergistic pathogenic effects of co-infection of subgroup J avian leukosis virus and reticuloendotheliosis virus in broiler chickens. Avian Pathol (2015) 44:43-9. doi:10.1080/ 03079457.2014.993359

38. Ellis CL, Ma ZM, Mann SK, Li CS, Wu J, Knight TH, et al. Molecular characterization of stool microbiota in HIV-infected subjects by panbacterial and order-level 16S ribosomal DNA (rDNA) quantification and correlations with immune activation. J Acquir Immune Defic Syndr (2011) 57:363-70. doi:10.1097/QAI.0b013e31821a603c

39. Brenchley JM, Price DA, Schacker TW, Asher TE, Silvestri G, Rao S, et al. Microbial translocation is a cause of systemic immune activation in chronic HIV infection. Nat Med (2006) 12:1365-71. doi:10.1038/nm1511

40. Hunt PW, Landay AL, Sinclair E, Martinson JA, Hatano H, Emu B, et al. A low $\mathrm{T}$ regulatory cell response may contribute to both viral control and generalized immune activation in HIV controllers. PLoS One (2011) 6:e15924. doi:10.1371/journal.pone.0015924

41. Marchetti G, Cozzi-Lepri A, Merlini E, Bellistrì GM, Castagna A, Galli M, et al. Microbial translocation predicts disease progression of HIV-infected antiretroviral-naive patients with high CD4+ cell count. AIDS (2011) 25:1385-94. doi:10.1097/QAD.0b013e3283471d10

Conflict of Interest Statement: The authors declare that the research was conducted in the absence of any commercial or financial relationships that could be construed as a potential conflict of interest.

The reviewer PR and handling editor declared their shared affiliation.

Copyright $\odot 2017$ Cui, Wang, Wang, Li, Wang and Zhao. This is an open-access article distributed under the terms of the Creative Commons Attribution License (CC BY). The use, distribution or reproduction in other forums is permitted, provided the original author(s) or licensor are credited and that the original publication in this journal is cited, in accordance with accepted academic practice. No use, distribution or reproduction is permitted which does not comply with these terms. 